\title{
THEMEN
}

\author{
Thomas Trenczek
}

\section{Restorative Justice in Neuseeland - Conferencing im Rahmen des strafrechtlichen Verfahrens zwischen Tradition und Moderne}

\section{Abstract}

Während meiner bisherigen Forschungsaufenthalte in Australien hatte ich bereits die Gelegenheit, die Praxis der Conference-Verfahren mit Jugendlichen in Australien kennenzulernen. ${ }^{1}$ Restorative Justice und Conferencing werden freilich häufig mit Neuseeland (NZ) und dessen indigener Bevölkerung in Verbindung gebracht. Heute wird der RJ-Ansatz in NZ bei Konflikten an Schulen, am Arbeitsplatz, im Gemeinwesen und insb. zur Unterstützung bei familieninternen Konflikten sowie auch in strafrechtlichen Verfahren gegen Jugendliche und Erwachsene genutzt. Die Implementation in das (jugend)strafrechtliche Verfahren hat allerdings in der Praxis zu erheblichen Verkürzungen der RJ-Konzeption geführt. Der Verfasser hatte während seines letzten Forschungsaufenthalts down under im Jahr 2011/12² die Gelegenheit, an der Jahrestagung von Restorative Justice Aotearoa ${ }^{3}$ (RJA) teilzunehmen und einige Conferencing-Programme vor Ort zu besuchen. ${ }^{4}$ Der folgende Beitrag fasst diese Erfahrungen und einige kritische Reflexionen zusammen.

Restorative Justice (RJ) is widely been said to be based on indigenous conflict resolution traditions, especially of the Maori culture in New Zealand (NZ). Today, in NZ restorative

1 Trenczek ZJJ 2002, 393 ff..

2 Der Verfasser war von Sept. 2011 - Febr. 2012 Visiting Scholar an der Griffith University Law School, Brisbane (Aus.) und verbrachte davon zwei Monate auf Einladung der School of Social and Cultural Studies, Massey University, Auckland in Neuseeland.

3 Name des nationalen RJ-Verbandes; „Aotearoa“ ist die Maori-Bezeichnung für Neuseeland und bedeutet etwa „Land der langen weißen Wolke“.

4 Für die mir entgegengebrachte Gastfreundschaft, den offenherzigen Diskurs und die dort gemachten Erfahrungen insb. in Fällen, an denen ich als teilnehmender Beobachter in einer Conference anwesend sein durfte, bin ich meinen Gastgebern zu großem Dank verpflichtet. Ebenso bedanke ich mich bei den Vertretern des neuseeländischen Justizministeriums, die mich eingeladen hatten, meine Erfahrungen mit ihnen zu reflektieren und über die Unterschiede zu europäischen Programmen zu diskutieren. 
justice procedures are used with relation to workplace disputes, within schools and community affairs, but also within the juvenile and criminal justice procedure. The implementation of RJ idea within the criminal justice system however has produced problems and shortcomings with regard to the RJ philosophy. During his last research visit down under in 2011/12 the author has had the opportunity to visit the annual conference of Restorative Justice Aotearoa as well as several RJ providers in NZ. This paper summarizes and reflects the collected data.

Keywords deutsch: Restorative Justice, Conferencing, Reintegrative Shaming, TäterOpfer-Ausgleich; Neuseeland

Keywords englisch: Restorative Justice, Conferencing, Reintegrative Shaming, VictimOffender-Mediation; New Zealand

\section{A. Restorative Justice und Conferencing}

\section{Begriffsklärung und Besonderheiten des Strafverfabrens in Neuseeland}

Der Begriff Restorative Justice (RJ) wird auf unterschiedlichen Ebenen mit unterschiedlichen Inhalten verwendet. Zunächst bezieht er sich auf ein die traditionelle Vergeltungslogik (retributive justice) und Strafphilosophien überwindendes Gerechtigkeitskonzept. Danach soll das aus der Begehung von Unrecht (nicht nur strafrechtlich relevanten Verhaltens) erfahrene Leid soweit wie möglich ausgeglichen und die als gerecht akzeptierte Ordnung in einer sozialen Gemeinschaft (wieder) hergestellt werden (to restore justice). ${ }^{5}$ Innerhalb dieses auf Ausgleich und Wiedergutmachung gerichteten Ansatzes finden sich eine Vielfalt unterschiedlicher Theorie- und Praxismodelle unterschiedlicher Reichweite. ${ }^{6}$ In Europa wurde es vor allem bekannt als Gegenmodell zur herrschenden Strafrechtsdoktrin im Hinblick auf die Bearbeitung strafrechtlich relevanten Verhaltens. ${ }^{7}$

Mit RJ ist also durchaus Unterschiedliches gemeint. So differenziert auch das neuseeländische Justizministerium zwischen der (andersartigen) Idee über Kriminalität zu denken und dem spezifischen Verfahren im Umgang mit Straftaten. Es gehe im Wesentlichen um ein Verfahren, "whereby all the parties with a stake in a particular offence come together to resolve collectively how to deal with the aftermath of the offence and its implications for the future. " 8 Damit verbunden sind in NZ eine Reihe höchst unterschiedlicher Zielsetzungen, u.a. die Verantwortungsübernahme durch den Täter, die Berücksichtigung von Opferinteressen ebenso wie öffentlicher Interessen, insb. der Resozialisierung und Förderung der Legalbewährung von Straftätern. Das in Neuseeland vorherrschende

5 Trenczek 2013a; Zehr 1985; ders, 2002.

6 Domenig 2011, 24 ff.; Trenczek 2013a.

7 Pelikan/Trenczek 2006, $63 \mathrm{ff} . ;$.

8 NZ Ministry of Justice, Appendix zu Restorative Justice Provisions in the Sentencing Act 2002 (http://www.justice.govt.nz/) mit Bezug auf Marshall 1996, 5.

NK 25. Jg. 3/2013 
RJ-Modell ist dabei die Integration des Conferencing Verfahrens im Rahmen der Strafzumessung (s. hierzu im Einzelnen B.).

Zum besseren Verständnis der neuseeländischen RJ-Praxis sei hier nur kurz auf zwei wesentliche Unterschiede zum deutschen Strafverfahren hingewiesen. Das Strafverfahren in NZ folgt im Wesentlichen der Tradition des Rechts angelsächsischer Prägung (common law), dessen Kennzeichen die Zweiteilung des Hauptverfahrens ist, einerseits zur Schuldfeststellung entweder (in aller Regel) aufgrund eines Schuldeingeständnisses (sog. guilty plea) mitunter nach entsprechenden Verhandlungen zwischen Justizbehörden und Beschuldigten (sog. plea bargaining) oder (weitaus seltener) nach einem Gerichtsverfahren (ggf. mit einer Geschworenenjury) sowie andererseits in einem zweiten Verfahrensabschnitt der Anhörung zur Festlegung der Sanktion (sentencing hearing). Zudem folgt das Strafverfahren des Common Law - anders als in Deutschland - nicht dem strengen Legalitätsprinzip. Insbesondere im Bereich der leichten und Bagatellkriminalität verfügt auch die Polizei über einen erheblichen Ermessensspielraum und beendet ein Verfahren häufig mit einer Verwarnung oder der Initiierung bestimmter Leistungen, ggf. einer Wiedergutmachungsweisung bzw. einer Aufforderung, an einem RJVerfahren teilzunehmen.

\section{Historische Entwicklung und indigene Wurzeln}

Im Unterschied zur konflikttheoretisch und kriminologisch geprägten RJ-Diskussion in Europa ${ }^{9}$ hat sich das Conferencing-Verfahren in NZ eher aus der Praxis entwickelt. Eine Verbindung zur Restorative Justice Idee wurde erst später hergestellt. ${ }^{10}$ Nachdem bereits 1989 die sog. Family Youth Conference (FYC) $)^{11}$ zusammen mit der Einrichtung von besonderen Jugendgerichten (Youth Court) durch das Jugend- und Familiengesetz (Children, Young Persons, and Their Families Act - CYPFA) eingeführt wurde, ${ }^{12}$ haben Judge McElrea und einige überzeugte Mitstreiter 1994 die Einführung des Conferencing auch in strafrechtlichen Verfahren (gegen Erwachsene und als solche behandelte Jugendliche) gefordert. In einem ersten Pilotversuch förderte das Justizministerium $1995 \mathrm{zu}-$ nächst drei Projekte in Hoani Waititi Marae (West Auckland), Timaru and Rotorua. Im Jahr 2002 wurden Conference-Verfahren als sog. Community Conference auch in das

9 Vgl. z.B. Christie 1977, 5 ff.; Hanak/Stehr/Steinert 1989.

10 Maxwell/Lin 2007; McElrea J.C.C.L 2011, 44-54.

11 Zur FYC siehe Maxwell/Morris 1993; Wiese 2010.

12 Damit hat sich Neuseeland ungeachtet der Gesetzesbezeichnung (Children, Young Persons, and Their Families Act) im Hinblick auf die Sozialkontrolle von Kindern (unter 14 Jahren) und Jugendlichen („young person“ 14 - 16 Jahren) von einem rein (sozial)rechtlich verfassten Welfare-Model (Kindeswohlorientierung, alleinige Zuständigkeit der Familiengerichte) verabschiedet. Gleichzeitig sollte nicht ein reines Strafrecht-(„Justice“)-Model praktiziert werden, sondern man erhoffte sich von der Einführung der FYC eine Versöhnung der beiden Systemmodelle. Ziel der FYC war vorrangig, die soziale Integration des jungen Menschen: "Itspurpose is to make such decisions, recommendations and plans as are thought to be necessary or desirable in relation to the child or young person in respect of whom the conference was convened." (Ministry of Justice: Family Group Conference 2013). 
allgemeine Strafrecht (NZ Sentencing Act 2002; Parole Act 2002; Victim Rights Act 2002 und Corrections Act 2004) eingeführt. Im Jahr 2011 wurden vom Justizministerium landesweit 23 gemeinnützige Träger zur Durchführung des Conference-Verfahrens mit einem Gesamtbudget von 2,55 Mio NZ\$ (ca. 1,5 Mio $€$ ) gefördert. Anfang 2013 wurde der Zuschuss auf insg. 3,05 Mio NZ\$ (ca. 1,8 Mio $€$ ) erhöht, mittlerweile ist die Zahl der geförderten Projekte allerdings auch auf 38 gestiegen, deren Förderung sich nach den vertraglich vereinbarten Fallzahlen richtet. ${ }^{13}$ Daneben gibt es eine Reihe weiterer, allerdings nicht vom Justizministerium geförderter RJ-Anbieter, die allein auf Selbstmelderfälle und eine entsprechende Finanzierungsstruktur (kommunale Mittel, Entgelt, Spenden, o.a.) angewiesen sind.

Ursprünglich wurde mit der Idee der „Conference“ versucht, die kulturellen Traditionen der Maori und Pacific Island Völker im Hinblick auf den strafrechtlichen Umgang mit Jugendkriminalität zu berücksichtigen und somit bei ihnen eine größere Akzeptanz des staatlichen Systems der Sozialkontrolle zu erreichen. Nach den Traditionen der Maori (wie anderer polynesischer Ethnien) betreffen Unrechtstaten nicht nur das unmittelbar verletzte Opfer, sondern die gesamte Großfamilie und Sippe (whänau), also die soziale Gemeinschaft beider, der Opfer wie der Täter. Da diese als solche geschädigt wurde, musste sie folgerichtig auch bei der Konfliktbearbeitung mit einbezogen werden, weshalb eine Gemeinschaftsversammlung („wänanga“ bzw. „bui“, engl. „conference“) einberufen wurde, an der abhängig von der Bedeutung der Sache die relevanten Angehörigen (insb. Entscheidungsträger) der (Groß-)Familie und Clans (whänau) bzw. des Stammes (hapi) der Betroffenen bzw. mehrere Stämme eines Volkes (iwi) teilnahmen. Entscheidungen wurden weitgehend im Konsens getroffen und kollektiv verantwortet. Die Betroffenen konnten sich dadurch der Unterstützung der Familie/Sippe sicher sein, die Inklusion ist nicht nur für den einzelnen, sondern für die Sippe existenziell, der Ausschluss (Exklusion) ist oft gleichbedeutend mit dem Tod. Deshalb basier(t)en die indigenen Stammesstrukturen auf einem starken Interesse nach sozialer Kohäsion, strengen Hierarchien und einer engen sozialen Kontrolle. Sie wurzeln in einer ausgeprägten Schamkultur, die nicht das einzelne Individuum, sondern das Kollektiv in den Mittelpunkt stellt. ${ }^{14}$

In den Versammlungen ging es zwar auch um Wiedergutmachung des Unrechts, des gebrochenen sozialen Friedens, sie dienten allerdings in erster Linie nicht dem materiellen oder emotionalen Wiedergutmachungsinteresse des unmittelbaren Opfers. Von Bedeutung ist, dass Kompensationen in ein kommunikatives System des Aushandelns und der Vermittlung zwischen den Großfamilien/Sippe eingebunden sind. Das Verfahren gleicht mitunter einer Zeremonie und beinhaltet auch spirituelle Elemente. Hand-

13 Nach den vom Justizministerium ausgegebenen Förderungsrichtlinien wird von den geförderten Programmen eine Mindestzahl von 30 Conferencing Verfahren im Jahr erwartet (Ministry of Justice: Restorative Justice Funding Framework July 2011, 12).

14 Demgegenüber ist für westlich-individualistische Gesellschaften und deren Rechtssysteme eine Schuldkultur kennzeichnend, die auf Ausgrenzung des Abweichenden, Vergeltung und letztlich Ausschluss (Exklusion) basiert. Zu den Unterschieden in der Scham- und Schuldkulturen vgl. Schirrmacher/Müller 2006.

NK 25. Jg. 3/2013 
lungsort war zumeist ein Marae, das zeremonielle Zentrum des Stammes. Der Ablauf der Conference orientiert sich an kulturellen Riten, am Status der Teilnehmenden und den entsprechenden Anordnungen von Redebeiträgen und nicht an der Logik der aus der modern-westlichen Kommunikations- und Konfliktforschung entwickelten Mediationsphasen. ${ }^{15}$

Ziel ist in diesen Systemen aber nicht die Konfliktlösung im westlich-modernen Sinn zwischen den unmittelbar betroffenen Konfliktparteien, Opfer und Täter, sondern die Herstellung von Harmonie und Gruppenstärke. Das Verfahren war nicht auf individuelle Schadenswiedergutmachung, sondern auf Versöhnung (reconciliation-kimipatero, kimi hamuti) im Kollektiv ausgerichtet. Nicht der Konsens zwischen zwei Konfliktparteien, sondern der Konsens im Kollektiv war für die Conference sinnstiftend. Es diente nicht in erster Linie den Interessen der verletzten unmittelbaren Opfer, sondern vor allem dem Schutz des Täters und seiner Sippe vor unkontrollierter (Blut-)Rache und Vergeltung. Denn gerade die Maoristämme waren nicht so friedliebend, wie sie bisweilen romantisch verklärt werden, vielmehr war die blutige Fehde zwischen Clans und Stämmen geradezu kennzeichnend für ihre Kultur. Die Kanalisierung der Konfliktaustragung diente damit vor allem dem Erhalt der indigenen Gesellschaft mit ihren Clan- und Stammesstrukturen. Vor diesem Hintergrund verwundert es nicht, dass im Informationsmaterial des neuseeländischen Justizministeriums auf die auch der Versöhnung zwischen den verschiedenen Stämmen dienenden Restorative Justice Elemente des für die Gründung der neuseeländischen Nation so wichtigen „Treaty of Waitangi“ zwischen der englischen Krone und den Chiefs von 540 Maori Stämmen hingewiesen wird.

\section{Reintegrative Shaming als konzeptionelle Basis von Restorative Justice?}

Auch in dem RJ-Ansatz geht es nicht nur um den individuellen Ausgleich zwischen Geschädigten und Verursacher, sondern auch um den Ausgleich der Störungen des Zusammenlebens in der sozialen Gemeinschaft. ${ }^{16}$ Der RJ-Konfliktbegriff bezieht Personen mit ein, die durch das Unrecht (nicht nur unmittelbar) betroffen sind, z.B. die Angehörigen, die Familie, die Mitbewohner, die Dorfgemeinschaft. In diesem Sinne knüpft die (moderne) RJ-Idee an historisch überlieferte Vorbilder bzw. die Regelungssysteme indigener Gemeinschaften an.

Der australische Kriminologe John Braithwaite hat unter dem Schlagwort des sog. „Reintegrative Shaming“ versucht, die Elemente der Scham-Kultur indigener Völker, insb. auch der Maori in NZ, in die Resozialisierungprogramme für junger Straftäter zu integrieren. ${ }^{17}$ Nach Braithwaite seien intakte Gesellschaften mit einem hohen Potential an Zusammenhalt und niedrigen Kriminalitätszahlen dadurch gekennzeichnet, dass sie bei einem („über die Stränge schlagenden“, rebellischen) Fehlverhalten ihrer jugendli-

15 Hierzu vgl. Mayer 2013, Rn 14.

16 Ausführlich Trenczek $2013 \mathrm{a}$.

17 Braithwaite 1989; ders. 1993, S. 39 ff.; Braithwaite/Mugford 1994; hierzu siehe Trenczek ZJJ 2002, 393 ff. sowie zuletzt Kersten ZJJ 2012, 169 ff. 
chen, heranwachsenden Mitglieder auf Erziehungsmethoden zurückgreifen, die von dem Jugendlichen auf der einen Seite Reue erwarten und gleichzeitig ein Angebot der Wiedereingliederung machen. Nicht ein Schuldspruch, sondern die empfundene Scham über das begangene Unrecht mache eine Wiedergutmachung erst möglich. Mit dem Prozess des „Reintegrative Shaming“ sollte die indigene Verantwortungs- und Versöhnungstradition für die moderne Gesellschaft nutzbar gemacht werden. Jugendliche sollen in einem selbst-reflexiven, reinigenden Läuterungsprozess ihr Fehlverhalten bekennen, Scham (shame) empfinden und Reue zeigen. Reue und Scham sind hierbei notwendige Bestandteile eines kathartischen Heilungsprozesses, ohne die eine wirkliche Wiederaufnahme in die Gemeinschaft (reintegration) nicht möglich sei.

Was den „reintegrative shaming“-Ansatz mit RJ verbindet, ist die hervorgehobene Bedeutung der Wiedergutmachung und der Anspruch auf Reintegration des Übeltäters in die soziale Gemeinschaft. Ebenso wenig sollen auf individueller (psychologischer) Ebene die positiven, entwicklungsfördernden Aspekte von echten Scham- und Reuegefühlen bezweifelt werden. Nicht harte Strafen, deren negative Auswirkungen empirisch hinreichend belegt sind, sondern „die Fähigkeit zur Perspektivenübernabme ist zusammen mit Bildung und der Kenntnis fremder Kulturen einer der stärksten Aggressionshemmer in allen menschlichen Gemeinschaften. Für die Chance zur Perspektivenübernabme müssen Möglichkeiten des Erlernens zur Verfügung gestellt werden. Obne die maßvolle Vermittlung von Schamgefüblen für ein Handeln, das Opfer hinterlassen hat und obne die damit zusammenbängende persönliche Übernabme von Verantwortung und die Bereitschaft zur Wiedergutmachung des entstandenen Schaden wird dies nicht möglich sein. Bestrafung ist keine Möglichkeit, dieses Ziel zu erreichen. "18 So richtig diese sanktionskritische Perspektive ist, der Shaming-Ansatz war nicht davor gefeit, in sein Gegenteil verkehrt zu werden.

Der nicht am Unrecht, sondern eher an einem individuellen Gefühl, einem Affekt, ansetzenden Schamkultur fehlt es zunächst auf gesellschaftlicher Ebene an der notwendigen Verbindlichkeit: „was verboten ist, ist weniger strittig als was beschämend wirkt. " ${ }^{19}$ Wesentlich ist aber vor allem, dass anders als im liebevollen Miteinader einer Familie (bzw. der Großfamilie und Sippe in indigenen Stammesstrukturen) es in unserer (modernen, individualisierten) Gesellschaft an den für reintegrative Verfahren ebenso wie für eine positive Schamkultur konstitutiven wertschätzenden, über die (Kern-)Familie hinausreichenden (öffentlichen) sozialen Beziehungen und Kollektiven fehlt. Braithwaite et al. differenzieren insoweit nicht hinreichend zwischen den unterschiedlichen Formen und Ebenen der Sozialkontrolle, sehen eine enge (auch polizeiliche) Kontrolldichte offenbar als unproblematisch positiv. Dem Ansatz liegt - nicht zuletzt aufgrund seiner Begrenzung auf abweichendes Verhalten von Jugendlichen - ein streng moralisches Grundverständnis von sozialer Kohäsion zugrunde, der sich mit einer rigiden Erziehungsideologie paart. Im Vordergrund steht nicht das „nicht herabwürdigende Vermitteln von Einsicht in eigenes Fehlverhalten“ und Förderung der „Perspektiven-

18 Kersten ZJJ 2012, 174.

19 Hilgers 2006, 20.

NK 25. Jg. 3/2013 
übernahme“ - beides zentrale Aspekte der RJ-Idee - sondern es geht um Beschämung und Buße. Von hier aus ist es nicht mehr weit zur Disziplinierung und Bestrafung. Braithwaite et al mögen stigmatisierende und herabwürdigende Beschämungstraditionen nicht im Sinn gehabt haben ${ }^{20}$ - das sei hier dahingestellt; die Praxis der Youth Conferencing Projekte in Australien, die sich auf den „reintegrative shaming“-Ansatz berief, scheint das offenbar nicht verstanden zu haben, denn dies ging einher mit der Ausweitung sozialer Kontrolle und führte nicht zu einer Abkehr, sondern zu einer Verstärkung der Vergeltungslogik. ${ }^{21}$

Schon der Fokus auf die Sozialkontrolle von jungen Menschen macht Braithwaites „reintegrative shaming“ zu einem Ansatz von nur begrenzter Reichweite, der trotz professioneller Marketingstrategien und seiner übermäßigen Rezeption im wissenschaftlichen Literaturkreislauf in der RJ-Praxis selbst in Australien kaum praktische Relevanz hat. Daran anknüpfende, z.T. naiv anmutende Transferentwürfe sind deshalb eher mit Vorsicht zu genießen. Was im Hinblick auf indigene Traditionen, (Scham-)Kultur und (kollektive) Strukturen sinnstiftend ist, lässt sich nicht so einfach in die westlich geprägte Schuldkultur und die entsprechenden - die individuelle Verantwortung des Menschen notwendigerweise voraussetzenden - Rechtsordnungen westlicher Gesellschaften übertragen.

\section{B. Conferencing im neuseeländischen Strafverfabren}

Conferencing-Verfahren werden in NZ außerhalb des Justizsystems bei Konflikten an Schulen, am Arbeitsplatz, im Gemeinwesen und insb. zur Unterstützung bei familieninternen Konflikten (insb. Erziehungsproblemen) genutzt sowie auch in (jugend-)strafrechtlichen Verfahren gegen Jugendliche und Erwachsene durchgeführt. Während RJVerfahren in strafrechtlichen Verfahren gegen Erwachsene (und als solche behandelte junge Menschen) vom Justizministerium administriert werden, ist das Sozialministerium (Ministry of Social Development) für die sog. „Family Group Conferences“ bei jugendlichen Beschuldigten ${ }^{22}(10-16$ Jahre) zuständig. Projektträger sind in aller Regel gemeinnützige und in der (lokalen) Gemeinde verankerte (local community-based providers) Stiftungen (trusts) oder Vereinigungen, teilweise kirchliche Organisationen oder Einrichtungen der indigenen Bevölkerung. ${ }^{23}$

20 So Kersten ZJJ 2012, 169.

21 Trenczek ZJJ 2002, $393 \mathrm{ff}$.

22 In NZ wird auch im Rahmen von Diversionprogrammen stets von „Offender“ gesprochen.

23 Ministry of Justice: Restorative justice providers contracted by the Ministry of Justice 2013 (http://www.justice.govt.nz); vgl. auch die Liste der RJA-Mitglieder unter http:// www.restorativejusticeaotearoa.org.nz. 


\section{Anwendungsbereiche und Ablauf des Conferencing-Verfabrens}

In Strafverfahren gegen Erwachsene kann eine Conference im Wesentlichen auf drei Ebenen des Verfahrens stattfinden; zum einen bereits nach dem ersten Polizeikontakt, insb. einer Festnahme (arrest), auf Zuweisung der Polizei als Diversionsmaßnahme, zweitens vor dem Sentencing Hearing sowie drittens auch nach der Strafzumessung als Teil der angeordneten Sanktion (Bewährungsauflage). In der Praxis werden die meisten Conference-Verfahren im Vorfeld des Sentencing-Hearing auf Zuweisung des Richters des lokalen District Courts (etwa vergleichbar mit einem Amtsgericht) nach einem guilty plea (Schuldeingeständnis) initiiert. Die Conference ist dann in der kurzen Zeit (i.d.R. 2-3 Wochen) bis zum Sentencing Hearing durchzuführen. Über den Inhalt und Verlauf der Conference wird ein Bericht angefertigt, der dem Gericht vor dem Sentencing Hearing zugehen soll (s.u.).

Während die Durchführung einer FYC im Jugendverfahren grundsätzlich obligatorisch und die Teilnahme des jungen Menschen zwingend (also nicht freiwillig) ist, hängt die Durchführung einer Conference bei Erwachsenen von der ausdrücklich als „freiwillig" bezeichneten (im strafrechtlichen $Z$ wangskontext ${ }^{24}$ wohl mehr oder weniger freiwilligen) Zustimmung des Beschuldigten ab. ${ }^{25}$ Voraussetzung für ein Conferencing ist - auch im Rahmen der polizeilichen Diversion - die Übernahme der Tatverantwortung durch den Täter. Aus diesem Grund wird eine Conference stets erst nach einem Schuldeingeständnis (guilty plea) durchgeführt.

Zwar sind formal keine Delikte aus dem Anwendungsbereich des Conferencing Verfahrens ausgenommen, dieser ist aber faktisch durch die Zuständigkeit der fallzuweisenden Instanzen beschränkt. So kommen im Rahmen der polizeilichen Diversion für eine Conference ausschließlich leichte Übertretungen und Vergehen (Ordnungswidrigkeiten und Bagatelldelikte) in Betracht und dies auch nur dann, wenn der Beschuldigte über keine (wesentlichen) Einträge im Register verfügt. Einige schwere Delikte (sog. "indictable offences") wie z.B. Mord, Vergewaltigung, Brandstiftung und schwere Drogendelikte unterliegen der Zuständigkeit des High Courts und erfordern eine Schuldfeststellung aufgrund eines gerichtlichen Verfahrens, womit eine pre-sentencing Conferencing nicht möglich ist. Für den Bereich dazwischen und damit für den Großteil des Deliktsspektrums sind die District Courts zuständig, insb. (Einbruchs-)Diebstahl (theft and burglary), Hausfriedensbruch, Straßenverkehrsdelikte sowie alle Körperverletzungsdelikte einschließlich häuslicher Gewalt. Letztere sind zwar formal nicht von einer Conference ausgenommen, wurden auf Empfehlung des Justizministeriums aber eher ausnahmsweise einbezogen. ${ }^{26}$ Festzustellen ist eine große Scheu, schwerere und Gewaltdelikte - obwohl nicht formal ausgeschlossen - im Rahmen einer Conference aufzuarbeiten, was insb. auf das politische Klima und die Forderung nach mehr strafrechtlicher

24 Hierzu Lindenberg/Lutz 2013.

25 Ministry of Justice (Best Practice) 2011, 12.

26 Ministry of Justice (Best Practice) 2011, 25; hierzu siehe auch Tisdall/Farmer et al. 2007; Rutledge, 2011.

NK 25. Jg. 3/2013 
Härte zurückgeführt wird. ${ }^{27}$ „Begründet“ wird dies zumeist mit einer mangelnden (allerdings nicht weiter definierten) Angemessenheit sowie mit der Verpflichtung, die Sicherheit (safety) der Beteiligten zu gewährleisten. Mittlerweile scheint das Justizministerium aufgrund positiver Erfahrungen im In- und Ausland die Einbeziehung der Fälle der häuslichen Gewalt allerdings positiver einzuschätzen und hat hierzu ganz aktuell entsprechende Standards herausgegeben. ${ }^{28}$ In der Praxis überwiegen bei den meisten Projekten einfache Delikte (sog. summary offences mit einer Strafandrohung von max. 3 Monate Haft; z.B. dangerous driving, cannabis possession) und sog. „moderately serious offences" wie Körperverletzung und Bedrohung sowie Einbruchsdiebstahl. ${ }^{29}$ Selbst sog. opferlose Delikte (wie Drogen- und Waffenbesitz, Verstöße gegen public order) werden mitunter bearbeitet, da insoweit stets die Community geschädigt sei.

Nachdem ein Fall dem örtlichen Projektträger zugewiesen wurde, überprüft der (hauptamtliche) Projektkoordinator die Geeignetheit des Falles (unter Berücksichtigung ggf. bestimmter Ausschlusskriterien) und nimmt sodann zunächst schriftlich und dann telefonisch Kontakt mit den betroffenen Beschuldigten und anschließend dem Opfer auf, um ihr grundsätzliches Einverständnis in ein Conferencing-Verfahren einzuholen. Ist dies der Fall, sind persönliche Vorgespräche mit beiden Parteien vorgesehen, in denen diese über das Verfahren, den konkrete Ablauf einer Conference, die Konsequenzen im Hinblick auf das Strafverfahren etc. informiert und Fragen beantwortet werden.

Geleitet werden die Conference dann stets von zwei (zumeist nicht hauptamtlich beschäftigten) als Facilitator (z.T. als Coordinator) bezeichneten Moderatoren, die - sofern das Programm vom Justizministerium gefördert wird - grundsätzlich eine vom Justizministerium verantwortete bzw. zugelassene Schulung vorweisen müssen; eine spezifische Grundprofession wird nicht vorausgesetzt. Diese Schulung umfasst zunächst ein Selbst-/Fernstudium in Hinblick auf vier Module (Restorative justice process and principles; Offence victim and offender issues; Working across cultures in restorative justice; The New Zealand criminal justice system and restorative justice). Hierzu erhalten die Bewerber ein vom Justizministerium herausgegebenes Trainingsmanual mit BegleitDVD. Die Bearbeitungszeit wird mit max. $8 \mathrm{Std}$. je Modul angegeben. Innerhalb ca. eines Monates müssen die Trainees zu jedem Modul eine Hausarbeit (assignment) einreichen. Wird diese als bestanden akzeptiert, nehmen die Trainees an einem fünftägigen Praxisschulungsblock teil, an den sich eine sechsmonatige Praxisphase unter Aufsicht (mentoring) und schließlich eine abschließende Überprüfung (assessment) durch externe, beim Justizministerium akkreditierte Prüfer anschließt, bevor eine zunächst auf zwei Jahre beschränkte Zertifizierung erteilt wird. ${ }^{30}$

27 McElrea 2011, 53: In New Zealand "there has been an unboly alliance between the media and most politicians to promote the illusion that punitive reactions promote community safety despite all the evidence to the contrary."

28 Ministry of Justice, Restorative justice standards for family violence cases, 2013.

29 McElrea 2011, 49: "However, other provider groups dealt mostly with less serious cases that the police were happy to see diverted through a community panel process."

30 Ministry of Justice, Restorative Justice Facilitator Induction Training and Accreditation (http://www.justice.govt.nz) [Abruf 02.02.2013]. 
Als Mediatoren bezeichnet sich keiner der Vermittler, gilt doch Mediation als Verfahren insb. aufgrund der notwendigen Ergebnisoffenheit als für strafrechtliche Konflikte unpassend. Freilich ist auch im europäischen Tat- bzw. Täter-Opfer-Ausgleich das Vermittlungsergebnis aus Gründen des Opferschutzes nicht völlig ergebnisoffen. ${ }^{31}$ Dennoch ist das konflikttheoretische Verständnis europäischer Mediatoren in der Tradition Nils Christies $^{32}$ für die neuseeländischen Praktiker (überwiegend) irritierend. Eine Konfliktklärung ist im Conferencing nicht intendiert, der Konflikt wird so nicht gesehen, zwingt doch das strafrechtliche Verfahren den Offender vor der Teilnahme an einer Conference notwendig zu einem formellen Schuldeingeständnis. Zudem haben die $\mathrm{Fa}$ cilitator die Funktion, nicht nur die Sicherheit aller Beteiligten und den fairen Ablauf des Verfahrens sicherzustellen, sondern auch, dass die (öffentlichen) Interessen der Community gewahrt werden und der Offender zur Verantwortung gezogen und weiteren Straftaten vorgebeugt wird. Diese dem strafrechtlichen Kontext geschuldete Rollenzuweisung unterscheidet die Facilitator letztlich von allparteilichen Mediatoren.

Üblicher Weise sollen an einer Conference neben dem Offender und Facilitator auch die geschädigten Opfer teilnehmen (hierzu siehe nachfolgend II.). Darüber hinaus wird auch die Einbeziehung weiterer Personen aus der Community empfohlen, was gerade ein Kennzeichen der Conference - im Unterschied zur Mediation - ausmacht. Allerdings ist der Begriff "Community“ - obwohl allgegenwärtig - nicht einheitlich definiert. ${ }^{33}$ Zum einen sind damit die persönlichen Unterstützer (Familienmitglieder, Freunde), andererseits aber auch sonstige Gemeindemitglieder oder weitere Personen gemeint, die durch die Straftat zumindest mittelbar betroffen („affected“) sind. Einige Programme haben hierfür ein Panel von engagierten Bürgern gebildet, von denen stets zwei oder mehrere Personen als Vertreter der Gemeinde (representatives of the community) an der Conference teilnehmen. Sog. „Professionals“, d.h. Polizeibeamte, Gerichts- und Bewährungshelfer (probation officers) oder Strafverteidiger können ebenfalls teilnehmen, allerdings „on a carefully prescribed basis“. Ihre Rolle sei es, ggf. Rat und Unterstützung zu leisten, nicht aber das Gespräch in einer Conference zu dominieren. ${ }^{34}$

Zwar wird die Conference grundsätzlich als nicht öffentliches Verfahren bezeichnet, was allerdings die Anwesenheit von weiteren Personen als Repräsentanten der Community nicht ausschließt. Die Vertraulichkeit der Gespräche ist also begrenzt, allerdings dürfen die Anwesenden die im Rahmen einer Conference erhaltenen Informationen Dritten gegenüber nicht offenbaren. Anders ist dies aber für anwesende Polizeibeamte und sogar die Facilitator, denn sie sind verpflichtet (die Beteiligten werden entsprechend darauf hingewiesen), im Rahmen eines Conferencing offenbartes strafrechtlich relevantes Verhalten an die Polizei zu melden..$^{35}$

31 International werden solche mediativen, aber nicht völlig ergebnisoffenen Verfahren mitunter „conciliation“ genannt. Zur begrenzten Ergebnisoffenheit auch in der zivilen Mediation Trenczek et al. 2013, Kap. 1.1.3.2.8, Rn $41 \mathrm{f}$.

32 Christie BJCrim 1977.

33 Zur Problematik des Begriffs Community vgl. Trenczek et al. 2013, Kap. 5.22, Rn. 4 ff.

34 Ministry of Justice (Best Practice) 2011, 14.

35 Ministry of Justice (Best Practice) 2011, 18.

NK 25. Jg. 3/2013 
Abb. 1: Ablauf des Restorative Justice Verfahrens (Informationsblatt RJ Nelson)

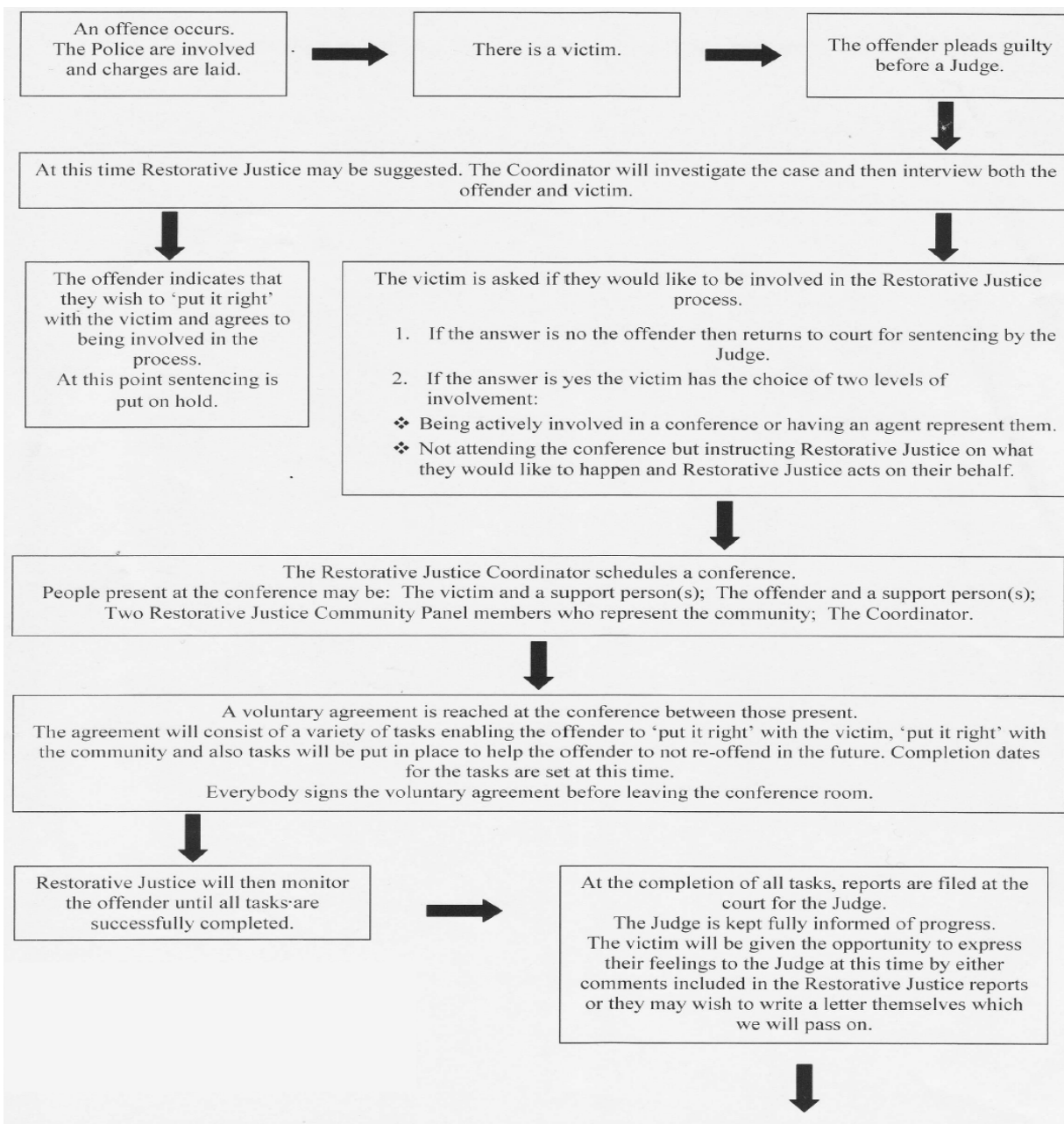

The offender returns to the Court and appears before the Judge for sentencing.

The Judge will consider the tasks undertaken and completed by the offender and all other written reports submitted for the final sentencing.

Als Ergebnis einer Conference werden konkrete Wiedergutmachungsleistungen des Täters erwartet, seien es finanzieller Schadensersatz, persönliche Wiedergutmachungsleistungen an das Opfer oder gemeinnützige Leistungen sowie ggf. weitere Verpflichtungen des Offenders im Hinblick auf sein Versprechen, sein Leben zu ändern. Diese sollen gleichzeitig eine „angemessene“ und „realistische“ Reaktion auf die Straftat darstel- 
len. ${ }^{36}$ Die Ergebnisse der Conference sollen laut Gesetz bei der abschließenden Verfahrens- bzw. Sanktionsentscheidung des Gerichts berücksichtigt werden (Sec. 8 und 10 Sentencing Act), wobei der strafmildernde Einfluss auf die Sanktionsentscheidung nach Auskunft der befragten Praktiker beträchtlich sein könne, was auch von einigen höchstrichterlichen Entscheidungen bestätigt wird. ${ }^{37}$ Allerdings ist es Sache der zuständigen Richter, zu prüfen, ob die Wiedergutmachungsleistung des Täters „aufrichtig und durchführbar" und ob das Opfer mit einer Berücksichtigung als strafmildernder Faktor einverstanden ist (Sec. 10, Sentencing Act 2002). Deshalb muss von den Facilitatoren über das Conferencing-Verfahren und dessen Ergebnis ein Bericht an das Gericht abgegeben werden, der neben anderen Faktoren die Grundlage für die abschließende Strafzumessungsentscheidung des Gerichts darstellt. ${ }^{38}$ In der Praxis wird damit durchaus sehr unterschiedlich umgegangen. Während einige Programme auf Wunsch der Richter ein ausführliches Inhaltsprotokoll der Conferencing erstellen, geben andere lediglich eine kurze Rückmeldung über Zeit und Ort sowie Ergebnis der Conference.

Die vom Gericht akzeptierten Ergebnisse des Conferencing werden von den Trägereinrichtungen überwacht und deren Erfüllung an das Gericht zurück gemeldet. Jede Abweichung vom Wiedergutmachungsplan muss dem Gericht gemeldet werden und kann zu einer gerichtlichen Anhörung und ggf. Sanktionierung führen (sec. 10 (4) und sec.110 Sentencing Act 2002).

\section{Die Einbeziehung und Rolle der Opfer}

Nach dem Victim Rights Act 2002 soll in Conference-Verfahren gegen Erwachsene grds. ein Treffen zwischen Opfer und Täter angeregt werden, wenn die Beteiligten hierzu bereit sind, entsprechende Ressourcen zur Verfügung stehen und ein Treffen angemessen ist. Die Freiwilligkeit der Teilnahme am Conference-Verfahren sowohl der betroffenen Geschädigten wie auch der Beschuldigten wird in allen offiziellen Publikationen besonders hervorgehoben. ${ }^{39}$ Erforderlich sei stets ein „informed consent“, eine bewusste Entscheidung aufgrund ausreichender Information und der Möglichkeit vorausgehender Rechtsberatung.

Zum besseren Verständnis der RJ-Praxis in NZ will ich darauf hinweisen, dass man hier zwischen der grundsätzlichen Bereitschaft, einem Conferencing Verfahren zuzustimmen, einerseits und der persönlichen Teilnahmebereitschaft und Anwesenheit in einer Conference andererseits unterscheidet. Während die Anwesenheit des Beschuldigten im Rahmen einer Conference zwingend ist, steht es Geschädigten frei, ob sie persönlich teilnehmen wollen oder nicht. So weist das Justizministerium ausdrücklich darauf hin: „While the offender's presence and participation in a restorative justice process is always required, victims should not be pressured to participate in the process or personally attend

36 Ministry of Justice (Best Practice) 2011, 17.

37 McElrea 2011, 50 m.w.N.

38 Ministry of Justice (Best Practice) 2011, 18.

39 Ministry of Justice (Best Practice) 2011, 12.

NK 25. Jg. 3/2013 
a conference. Although full participation by the victim is the ideal, some programmes allow restorative justice processes to proceed so long as the victim agrees to the process taken place and the victim's views are represented." 40

Letzteres, also die Einbeziehung der Opferperspektive, soll dann ggf. über ein schriftliches victim impact statement, Opferanwälte (victim support worker) oder gar die Facilitators erfolgen. Was sich hier ausnimmt als eine unverzichtbare und angemessene Berücksichtigung der Opferinteressen, insb. zu deren Schutz, hat sich in der Praxis mancherorts ins Gegenteil verwandelt. Mittlerweile wird in einigen Programmen die Einbindung der Geschädigten als zu zeitaufwendig und schwierig angesehen und auf deren Beteiligung bzw. aktive Einbeziehung verzichtet. Beschuldigte müssen sich stattdessen im Rahmen der Conference mitunter gegenüber einem Panel von ausgewählten Vertretern der lokalen Gemeinschaft verantworten, welches die angemessene Wiedergutmachungsleistung festsetzt, der der Offender zustimmen muss.

Die Struktur der staatlichen Finanzierung durch das Justizministerium - obwohl quantitativ deutlich höher als in anderen Ländern - trägt nicht dazu bei, diese Entwicklung zu verhindern. So erhalten die Programmträger für ein RJ-Verfahren im Rahmen der polizeilichen Diversion einen Pauschalbetrag von 700 NZ\$ (ca. $420 €$ ), unabhängig davon, ob das Opfer an dem Verfahren teilnimmt oder nicht. Im Rahmen einer Fallzuweisung durch das Gericht erhalten die Projekte einen Pauschbetrag von 900 NZ\$ (ca. $535 €$ ), sind die Opfer persönlich anwesend, erhöht sich der Betrag auf $1.400 \mathrm{NZ} \$ .^{41} \mathrm{Nach}$ Ansicht mancher Projektträger lohne allerdings dieser erhöhte Betrag nicht den Aufwand der mit der angemessenen Einbeziehung der Opfer verbundenen Verfahrensweise (insb. vorbereitende Gespräche), vor allem, wenn in einem Fall nicht nur ein, sondern mehrere Geschädigte betroffen sind.

In den Programmen, die sich um eine aktive Beteiligung der Opfer der Tat bemühen, wird viel Wert gelegt auf deren Begleitung durch Familienangehörige, persönliche Freunde oder sonstige Opferbeistände aus dem sozialen Umfeld (in Fällen häuslicher Gewalt wird dies als Standard angesehen ${ }^{42}$ ). Die Sicherstellung des persönlichen Sicherheitsgefühls (emotional and physical safety) der Beteiligten, insb. der durch die Tat Geschädigten, gilt als alles beherrschender Faktor der Verfahrensgestaltung. Manche Programmanbieter legen auch Wert darauf, dass der Beschuldigte sein Verhalten auch mindestens gegenüber einer Person aus seinem privaten Umfeld einräumt, um damit sicherzustellen, dass er seine Verantwortung auch gegenüber seinem sozialem Umfeld und nicht nur einem für ihn abstrakten Justizsystem übernimmt. Andererseits sollen diese Personen ihn im RJ-Verfahren als Unterstützer begleiten und helfen, zu seiner Verantwortung zu stehen.

Wenn und soweit die Geschädigten an einer Conference teilnehmen, sind sie in der ganz überwiegenden Mehrheit (sehr) zufrieden (51 bzw. 31\%) mit dem Verfahren und

40 Ministry of Justice (Best Practice) 2011, 13.

41 Ministry of Justice (Funding Framework) 2011, 10.

42 Ministry of Justice (standards for family violence cases) 2013, 25.

THEMEN 
würden eine Teilnahme weiter empfehlen. ${ }^{43}$ Im Wesentlichen sei das darauf zurückzuführen, dass sie gut über das Verfahren informiert und gut darauf vorbereitet worden seien, ihre Interessen und Sorgen Beachtung gefunden haben, der Facilitator sich gegenüber allen Beteiligten fair verhalten und auch nach der Conference den Kontakt zum Geschädigten aufrecht erhalten (follow-up meeting) sowie der Beschuldigte seine Wiedergutmachungsleistungen erbracht habe. Als Grund für die Teilnahme nannten die befragten Geschädigten vor allem das Bedürfnis nach Klärung im Hinblick auf die Tat sowie nach einer Entschuldigung. Der Wunsch nach materiellem Schadensersatz wurde deutlich seltener genannt. Leider fehlen Informationen von den Personen, die es abgelehnt haben, an einer Conference teilzunehmen.

Abb. 2: Reasons for attending the conference that were rated as very important (Ministry of Justice 2011)

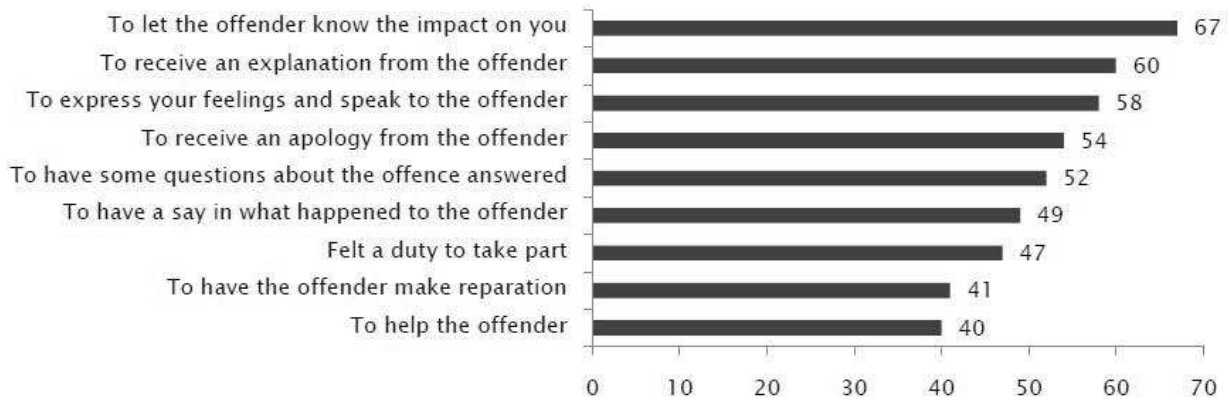

\section{Ergebnisse und Probleme in der Praxis der Conferencing Verfabren}

Im Jahr 2011 wurden von den 23 vom Justizministerium geförderten Projektträgern landesweit etwa 1800 Verfahren durchgeführt. ${ }^{44}$ Damit bleiben die Conferencing Verfahren auch in Neuseeland im Hinblick auf die Gesamtzahl der strafrechtlichen Verfahren eine Ausnahmeerscheinung, was Judge McElrea - einer der maßgeblichen Initiatoren des Conferencing - zum einen auf die Voraussetzung der freiwilligen Teilnahme der Beteiligten, die mangelnde Finanzierung der Projekte sowie auf die weithin straforientierte Einstellung der Justizpraxis und deren mangelnde Akzeptanz der RJ-Idee zurückführt. "The adversary ethos is so deeply imbedded in our legal structures, the legal profession, and the judges, who (in common law countries) are drawn from the profession, that restorative justice is continually pushed to the margins, despite the encouragement of the

43 Diese Zahlen basieren auf einer vom neuseeländischen Justizministerium veranlassten und im März 2011 durchgeführten telefonischen Befragung von (nur) 154 hierzu bereiten Geschädigten, die an einer Conference zwischen Sept. 2009 und Mai 2010 teilgenommen haben (Ministry of Justice: Victim satisfaction with restorative justice; Wellington 2011); vgl. auch Ministry of Justice 2007.

44 Information des Justizministeriums Stand Anfang 2012. Zu früheren im Rahmen der Evaluation des nationalen Pilotprojekts erhobenen Daten vgl. Ministry of Justice 2005.

NK 25. Jg. 3/2013 
legislators. ${ }^{45}$ Er spricht sich deshalb mittlerweile dafür aus, das Conferencing-Verfahren aus dem engen Korsett des strafrechtlichen Verfahrens herauszulösen und in außergerichtlich organisierte Community Justice Centers zu überführen. ${ }^{46}$

Das wohl größte Problem des Conferencing in NZ ist die abnehmende Zahl der an dem Verfahren teilnehmenden Opfer; nicht veröffentlichte, mir gegenüber erwähnte Zahlen schwanken zwischen (meistens) 50 und (selten) 75\% persönlicher Anwesenheit in Verfahren, in denen eine Conference (mit grds. Zustimmung der Geschädigten) stattfindet. ${ }^{47}$ Die Diskussion über die Stellung des Opfers im Rahmen des Conferencing Verfahrens hat innerhalb Restorative Justice Aotearoa zu Spannungen geführt. Auf der Mitgliederversammlung im Nov. 2011 wurde ein Antrag, nach dem Projekten, deren Fallbearbeitung eine Conferencing ohne Teilnahme der geschädigten Opfer vorsehen, die Akkreditierung zu verweigern ist, nur mit wenigen Stimmen Mehrheit abgelehnt.

In dieser Entwicklung spiegelt sich auch der starke Einfluss von Konzeption und Praxis der zuvor eingeführten Family Youth Conferences (FYC) wider (s.o. A. II.). In diesen steht ganz im Sinne der indigenen Traditionen einerseits und der bis dahin die Sozialkontrolle beherrschenden sozialrechtlichen Familienorientierung andererseits die Integration der jungen Menschen in die soziale Gemeinschaft im Vordergrund. Das Ziel der FYC ist nicht die Konfliktklärung und Berücksichtigung der Opferinteressen, sondern die zukünftige Entwicklung des jungen Menschen: "Their purpose is to make such decisions, recommendations and plans as are thought to be necessary or desirable in relation to the child or young person in respect of whom the conference was convened." ${ }^{48} \mathrm{Nicht}$ zuletzt deshalb werden in der Praxis die FYC auch ohne Opferbeteiligung als Panel bzw. wie vor einem (Erziehungs-)Komitee durchgeführt. Das Opfer ist insoweit - wenn man es ungeschminkt formuliert - nicht viel anders als im Strafverfahren lediglich Nebendarsteller und wird für die Erziehung des Jugendlichen instrumentalisiert. Das FYC entspricht damit mehr einem täterorientierten Resozialisierungsansatz mit erzieherischem Impetus und nicht dem Opferschutz-Ansatz der RJ-Idee.

Das Conference-Verfahren im Erwachsenenbereich scheint sich in dieselbe Richtung zu entwickeln. Von einem die Autonomie der Beteiligten - Opfer wie Täter - respektierenden Konfliktlösungsansatz ist hier auch nicht die Rede. Die Tat wird nicht - so wie in der europäisch-kriminologisch geprägten RJ-Bewegung - als Konflikt und dynamisches Geschehen angesehen. ${ }^{49}$ Es geht im Rahmen des Conferencing zwar auch um Wiedergutmachungsleistungen, vordringlich wird hierin aber - vergleichbar mit jeder anderen justiziellen Maßnahme - eine Sanktion zur Rückfallverhinderung gesehen. Hierzu veröffentlichte das neuseeländische Justizministerium nach 2005 im Jahr 2011 die Ergebnisse einer zweiten Vergleichsstudie, nach der an einer Conference teilnehmende Of-

45 McElrea 2011, $53 \mathrm{f}$.

46 McElrea 2011, 54.

47 Hinzurechnen muss man die Fälle, in denen die betroffenen Opfer das Conferencing-Verfahren grundsätzlich ablehnt haben.

48 Ministry of Justice: Family Group Conference (2013).

49 Vgl. z.B. Christie 1977, 5 ff.; Hanak/Stehr/Steinert 1989. 
fender eine um $20 \%$ niedrigere Rückfallquote aufwiesen. ${ }^{50}$ Hierin liegt - neben aller (durchaus ernst gemeinten) Rhetorik im Hinblick auf die stärkere Berücksichtigung der Opferinteressen - das eigentliche Interesse der Justizverwaltung und Rechtspolitik und - angesichts der immensen Kosten des Freiheitsentzugs ${ }^{51}$ - der Grund für die staatliche Förderung aus dem Justizbudget. Dieser Perspektive können sich auch die ConferenceProjekte nicht entziehen. ${ }^{52}$

\section{Reflexion der Erfabrungen und Ausblick}

Das in Neuseeland praktizierte Conferencing Verfahren unterscheidet sich von anderen RJ-Ansätzen, insb. der Vermittlung im Hinblick auf einen außergerichtlichen Tat- bzw. Täter-Opfer-Ausgleich (Victim-Offender Mediation) in Deutschland. Auch in Neuseeland ist der Versuch der Integration traditioneller Konfliktregelungsverfahren in das angelsächsisch geprägte Rechtssystem nicht ohne Spannungen und Reibungsverluste verlaufen. Projektverantwortliche mit indigenem Hintergrund haben mich zudem darauf hingewiesen, dass das offiziell im Rahmen des Strafverfahrens praktizierte Conferencing Verfahren nur noch wenig mit ihrer indigenen Kultur und Tradition zu tun habe, diesem aber den Anstrich kultureller Sensibilität gebe. Mit der Implementation in das staatliche Justizsystem hat sich das Conferencing-Verfahren in Neuseeland offenbar von seinen indigenen Vorläufern entfernt.

Gelernt für die eigene Praxis habe ich vor allem in der konkreten Begegnung und in dem offenen Austausch mit engagierten Praktikern sowie den Erlebnissen in den Conference-Verfahren mit Opfern und Beschuldigten. Als ganz konkrete, praktische Lernerfahrung konnte ich die positiven Wirkungen der stärkeren Einbindung von Unterstützerpersonen auf beiden Seiten, für Opfer und Beschuldigte, erfahren. Dies erfordert auf Seiten der Vermittler eine erhöhte Sensibilität für die Bedürfnisse der Betroffenen und gleichzeitig vermehrte Aktivität, geeignete Unterstützer zu finden und zu motivieren, an dem Verfahren teilzunehmen. Der Aufwand scheint sich zu lohnen, soweit ich dies atmosphärisch und in der Reflexion der von mir besuchten Conferencing Verfahren beurteilen kann.

Kennzeichen der neuseeländischen Praxis ist ein hohes Maß an Engagement und Pragmatismus, aus Sicht eines in der europäischen Diskussionskultur sozialisierten Gastes allerdings zum Preis des Verzichts auf eine konflikttheoretische Debatte und damit der Gefahr einer mangelnden konzeptionellen Klarheit. So faszinierend die indigenen Wurzeln des Conferencing Verfahren und die nur zum Teil noch sichtbaren Relikte traditioneller Konfliktregelung auch sind, aufgrund der zuvor beschriebenen Erfahrungen beobachte ich die Einbindung des Conferencing in das Strafzumessungsverfahren im

50 Ministry of Justice (Re-offending Analysis) 2011; Ministry of Justice (Two Year Follow-up of Reoffending) 2005.

51 Neuseeland hat nach den USA und Südafrika eine der höchsten Inhaftierungsraten im weltweiten Vergleich.

52 Vgl. McElrea 2011, 53; Rutledge 2011.

NK 25. Jg. 3/2013 
Hinblick auf Umsetzung der RJ-Idee mit Sorge. Auch wenn sich die Mitarbeiter der Conferencing-Programme in der täglichen Arbeit mit Beschuldigten und Opfern engagiert bemühen, dem RJ-Ausgleichsgedanken Geltung zu verschaffen, müssen sie letztlich den Erwartungen des Justizsystem und den strafrechtlichen Interessen Tribut zollen. Die Bedürfnisse der geschädigten Opfer werden im Rahmen des Conferencing zwar in einem weit stärkeren Maße beachtet als im traditionellen Strafverfahren, gemessen am RJ-Anspruch kommen die Opferinteressen aber wohl doch etwas zu kurz. Conferencing kann sich offenbar nicht über die Berücksichtigung der Opferinteressen legitimieren, sondern erwartet wird eine verbesserte Legalbewährung der teilnehmenden Straftäter.

Das ist im doppelten Sinne problematisch, denn zum einen sind gerade in der Rückfallforschung die mangelnden Erfolge und negativen Auswirkungen traditioneller, insb. in NZ relativ häufig verhängter freiheitsentziehender Sanktionen empirisch hinreichend belegt, zum anderen wird dadurch das Opfer für die Zwecke einer sich perpetuierenden Sicherheitslogik instrumentalisiert. Denn offenbar scheint sich nichts an der - jenseits der Verbalrhetorik - mangelnden Akzeptanz des Conferencing durch die Justiz zu ändern. Conferencing in NZ, eingebunden in das strafrechtliche Entscheidungsprogramm, bleibt eher der Sanktionslogik (bzw. systemtheoretisch formuliert: dem Eigeninteresse der Strafjustiz) verhaftet und nicht vorrangig dem RJ-Ansatz verpflichtet. ${ }^{53}$

Ungeachtet der angelsächsisch dominierten Fachdiskussion haben die ConferencingModelle in Europa bislang keine wesentliche Praxisrelevanz entwickeln können. Hier, insb. im deutschsprachigen Raum, ist die Vermittlung (Mediation) in strafrechtlich relevanten Konflikten im Rahmen eines Tat- bzw. Täter-Opfer-Ausgleichs der vorherrschende Anwendungsbereich der „Restorative Justice“-Idee. ${ }^{54}$ Freilich ist die Begrenzung der RJ-Praxis auf strafrechtliches Unrecht lediglich der konkreten Implementation des Ausgleichsgedankens in das Strafrechtssystem geschuldet. Restorative Justice versteht sich demgegenüber - worauf bereits eingangs hingewiesen wurde - als umfassendes, traditionelle Strafphilosophien überwindendes Gerechtigkeitsparadigma, nach dem das aus der Begehung von Unrecht (nicht nur strafrechtlich relevanten Verhaltens) erfahrene Leid soweit wie möglich ausgeglichen werden soll. Auch in NZ werden ConferenceVerfahren vor allem im Schulbereich, bei Konflikten im Gemeinwesen oder am Arbeitsplatz angewendet. Der Transfer in das strafrechtliche Kontrollsystem muss notwendigerweise zu einer Beschneidung wesentlicher Elemente der RJ-Idee führen.

Es verwundert nicht, dass sich insbesondere auch einige Gründerväter der Conferencing-Idee dafür aussprechen, das Conferencing-Verfahren aus dem engen Korsett des strafrechtlichen Verfahrens herauszulösen und in ein der RJ-Idee besser entsprechendes System außergerichtlich organisierter Community Justice Centers zu überführen. ${ }^{55}$ Auch in Deutschland hat die Rückbesinnung auf die Wesensmerkmale der RJ-Idee bereits einige Initiativen (z.B. Waage Hannover) dazu bewogen, sich nicht auf den TOA zu beschränken, sondern ihr Vermittlungsangebot inhaltlich breiter und gemeinwesen-

53 Grundsätzlich zu dieser Problematik Trenczek CJR 2002, $23 \mathrm{ff}$.

54 Pelikan/Trenczek 2006, 63 ff.; Trenczek 2013c.

55 McElrea (Fn. 10), 53 f.; vgl. Trenczek CJR 2002, 33 f..

THEMEN 
nah auszugestalten. Im internationalen Raum wird mittlerweile nicht mehr nur von RJ, sondern von Restorative Practice gesprochen, um sich von der vereinnahmenden Definitionsmacht des Strafrechts zu lösen. ${ }^{56}$

\section{Literatur}

Braithwaite, J. (1989) Crime, Shame and Reintegration, Sydney

ders. (1993) Juvenile Offending: New Theory and Practice; in: Atkinson/Gerull (eds.), National Conference Juvenile Justice. Conference Proceedings No. 22, Australian Institute of Criminology; Canberra/Aus

Braithwaite.J./Mugford, S. (1994) Conditions of Successful Reintegration Ceremonies: Dealing with Juvenile Offenders; British Journal of Criminology, vol. 34 (2), S. 139-171

Christie, N. (1977) Conflicts as Property, British Journal of Criminology, S. 1 - 15

Domenig, C. (2011) Restorative Justice, TOA-Infodienst Nr. 41/2011, S. 24 (01-10)

Hanak, G./Stebr, S./Steinert,S. (1989) Ärgernisse und Lebenskatastrophen. Über den alltäglichen Umgang mit „Kriminalität", AJZ-Verlag, Bielefeld

Hilgers, M. (2006) Scham, Gesichter eines Affekts, 3. Auf. Göttingen

Kersten, J. (2012) „Restorative Justice“ - Innovative Ansätze im Umgang mit Konflikten und Gewaltereignissen, Zeitschrift für Jugendkriminalrecht und Jugendhilfe 2012, S. 169 $-174$

Lindenberg, M./Lutz, T. (2013) Soziale Arbeit in Zwangskontexten; in Ak KrimSoz (Hrsg.) Kriminologie und Soziale Arbeit, Weinheim (im Druck)

Marshall, T. (1996) Restorative Justice: An Overview. Home Office, London/UK 1996, 5

Maxwell, G./Liu, A. (2007) Restorative Justice and Practices in New Zealand; Wellington/NZ

Maxwell, G./Morris, A. (1993) Family, victims and culture: youth justice in New Zealand. Wellington/NZ

56 Trenczek 2013b, 428; Wachtel 2011.

NK 25. Jg. 3/2013 
McElrea, F. (2011) Twenty years of restorative justice in New Zealand: reflections of a judicial participant; Journal of Commonwealth Criminal Law (J.C.C.L) vol. 1 (2011), S. $44-54$

Mayer, C.-H. (2013) Kulturpsychologische und ethnologische Einsichten; in Trenczek et al (Hrsg.), Mediation und Konfliktmanagement, 2013, Kap. 2.3, S. 86 - 91

Ministry of Justice (2005) Evaluation of the Court-Referred Restorative Justice Pilot: Technical Report - May 2005; Wellington/NZ

Ministry of Justice: (2005) New Zealand Court-Referred Restorative Justice Pilot: Two Year Follow-up of Reoffending, Wellington/NZ

Ministry of Justice (2007) Restorative justice: the views of victims and offenders, Wellington/NZ

Ministry of Justice (2011) Restorative Justice. Best Practice in New Zealand; Wellington/ NZ

Ministry of Justice (2011) Restorative Justice Funding Framework Wellington/NZ

Ministry of Justice (2011) Victim satisfaction with restorative justice; Wellington/NZ

Ministry of Justice (2011): Re-offending Analysis for restorative justice cases 2008 and 2009, Wellington/NZ

Ministry of Justice (2013) Restorative justice standards for family violence cases, 2013; Wellington/NZ

Ministry of Justice (2013) Family Group Conference [http://www.justice.govt.nz/ courts/youth/about-the-youth-court/family-group-conference, Febr. 2013]; Wellington/NZ

Ministry of Justice (2013) Restorative Justice Facilitator Induction Training and Accreditation; http://www.justice.govt.nz; Wellington/NZ

Pelikan, C./Trenczek, T. (2006) Victim offender mediation and restorative justice - The European landscape, in Sullivan, D./Tifft, L. (eds.) Handbook of Restorative Justice: A Global Perspective; Taylor and Francis, London (UK), S. 63-90

Rutledge, M. (2011) Are we working on the edge - Comparative quantitative analysis of restorative justice within a family violence context; Paper presented at the RJA-Conference, Wellington Nelson Nov. 2011 
Schirrmacher, T./Müller, K. (2006) Scham- und Schuldorientierung in der Diskussion. Kulturantropologische, missiologische und theologische Einsichten, Nürnberg/VTK

Tisdall, M./Farmer, S. et al. (2007) Review of the use of restorative justice in family violence cases in Rotorua district, Ministry of justice, Wellington/NZ

Trenczek, T. (2002) Victim-offender-reconciliation: The danger of cooptation and a useful reconsideration of law theory; Contemporary Justice Review (CJR) 2002, S. 23-34

ders. (2002) TOA mit erhobenen Zeigefinger, Zeitschrift für Jugendkriminalrecht und Jugendhilfe (ZJJ) 2002, 393-399

ders. (2013a) Restorative Justice, in AK KrimSoz (Hrsg.) Kriminologie und Soziale Arbeit; Weinheim (2013; im Erscheinen)

ders. (2013b), Beyond Restorative Justice to Restorative Practice; in Cornwell et al. (eds.), Civilizing Criminal Justice; Hook/Hampshire: Waterside Press 2013, S. 409 - 428

ders. (2013c) Restorative Justice in der Praxis: Täter-Opfer-Ausgleich und Mediation in Deutschland; in: TOA-Servicebüro, Köln (Hrsg.) Restorative Justice-Reader sowie TOA-Magazin 3/2013 (im Druck)

Trenczek, T./Berning, D./Lenz, C. (Hrsg.) (2013) Mediation und Konfliktmanagement - Handbuch; Baden-Baden

Wachtel, T. (2011) Restorative Practices. Creating a Unified Strategy for Democratizing Social Care, Education and Criminal Justice; paper presented on the 2011 European Congress on the Family Group Conference in Utrecht, the Netherlands, October 19-21, 2011

Wiese, K. (2010) Family Group Conferencing - Mehr gemeinschaftliche und familiäre Verantwortungsübernahme im Jugendstrafrecht? Frankfurt

Zebr, H. (1995), Retributive Justice - Restorative Justice, Elkart/USA

ders. (2002) Changing Lenses. A new focus on Crime and Justice, Harald Press/Scottdale (USA)

Prof. Dr. iur. Thomas Trenczek, M.A.; eingetragener Mediator (BMJ, Wien) (BMWA) (AMA); lehrt u.a. Jugend-, und Strafrecht, Kriminologie und Mediation/Konfliktmanagement an der Ernst Abbe Hochschule Jena; er ist Mitbegründer und 1. Vors. der Waage Hannover e. V., und des SIMK Hannover (BMWA-Ausbildungsinstitut $\left.{ }^{\circledR}\right)$.

www.simk.net / mediation@trenczek.net. 